\title{
Capilaroscopia Periungueal pode Sugerir Atividade de Doença Pulmonar na Esclerose Sistêmica ${ }^{(*)}$
}

\author{
Nailfold Capillaroscopy can Predict Active Pulmonary \\ Involvement in Systemic Sclerosis
}

\author{
Markus Bredemeier ${ }^{(1)}$, Ricardo Machado Xavier ${ }^{(2)}$, Karina Gatz Capobianco ${ }^{(3)}$, Vicente Gregório Restelli ${ }^{(4)}$, \\ Luis Eduardo Paim Rohde ${ }^{(5)}$, Antônio Fernando Furlan Pinotti ${ }^{(6)}$, Eduardo Hennemann Pitrez ${ }^{(7)}$, \\ Marcelo Vasconcelos Vieira ${ }^{(7)}$, Maria Ângela Fontoura ${ }^{(8)}$ e João Carlos Tavares Brenol ${ }^{(9)}$
}

\section{RESUMO}

Objetivo: Estudar a associação entre alterações capilaroscópicas e lesões em órgãos-alvo na esclerose sistêmica (ES). Métodos: Noventa e um pacientes com ES foram avaliados através de entrevista, exame fisico, capilaroscopia periungueal (CPU), sorologia, provas de função pulmonar, cintilografia de trânsito esofágico, ecocardiografia com Doppler e tomografia computadorizada de alta resolução pulmonar (TCAR). A deleção capilar na CPU foi avaliada usando o escore de deleção vascular de Lee; consideraram-se alterações severas na CPU um escore médio de deleção $\geq 1$ ou número médio de megacapilares por dedo $\geq 1$ para fins de análise. Hipertensão arterial pulmonar (HAP) foi definida como pressão sistólica na artéria pulmonar $\geq 40 \mathrm{mmHg}$. Resultados: Pacientes com alterações capilaroscópicas severas apresentaram maior prevalência de áreas de opacidades em vidro-fosco (OVF) $(\mathrm{P}=0,016)$, redução da capacidade difusional pulmonar $(\mathrm{P}=0,026)$ e disfunção esofágica $(\mathrm{P}=0,001)$. HAP ocorreu somente em pacientes com alterações severas na $\mathrm{CPU}(\mathrm{P}=0,114)$. Naqueles com duração de doença $\leq 5$ anos, OVF estavam presentes em 14 de 19 pacientes com alterações severas na CPU, mas não estavam presentes nos 8 pacientes com alterações capilaroscópicas leves ou ausentes $(\mathrm{P}<0,001)$. Nesse subgrupo, nenhuma outra variável clínica ou laboratorial associou-se à presença de OVF. O uso de curvas ROC mostrou uma boa capacidade da CPU em

\begin{abstract}
Objective: To evaluate the association of capillaroscopic alterations with end-organ damage in systemic sclerosis (SSc). Methods: Ninety-one SSc patients were studied through interview, physical examination, nailfold capillary microscopy (NCM), serology, pulmonary function tests, esophageal transit scintigraphy, Doppler echocardiography and pulmonary highresolution computed tomography (HRCT). Capillary loss on NCM was evaluated using the avascular score (AS); patients with mean $A S \geq 1$ or mean number of megacapillaries per finger $\geq 1$ were considered to have severe capillaroscopic alterations for analytical purposes. Pulmonary arterial hypertension (PAH) was defined as systolic pulmonary arterial pressure $\geq 40 \mathrm{mmHg}$. Results: Patients with severe NCM alterations had greater prevalence of ground-glass opacities (GGO) areas $(P=0.016)$, reduced pulmonary diffusing capacity $(P=0.026)$ and esophageal dysfunction $(P=0.001)$. PAH was present only in patients with severe NCM abnormalities $(P=0.114)$. In those with disease duration $\leq 5$ years, GGO were present in 14 of 19 patients with severe NCM alterations, but were absent in all patients $(n=8)$ with no or mild NCM alterations $(P<0.001)$. In this subgroup, no other clinical or laboratory variable was associated with the presence of GGO. ROC curves showed a good ability of NCM
\end{abstract}

\footnotetext{
* Trabalho realizado no Serviço de Reumatologia do Hospital de Clínicas de Porto Alegre, da Universidade Federal do Rio Grande do Sul (HCPA/UFRGS). Publicado J Rheumatol 31(2):286-94,2004. Recebido em 08/1 1/03. Aprovado, após revisão, em 26/01/04.

1. Médico Reumatologista, Mestrando em Clínica Médica, HCPA/UFRGS.

2. Professor Adjunto, PhD em Imunologia, Médico Reumatologista, Serviço de Reumatologia, HCPA/UFRGS.

3. Médica Reumatologista, Doutoranda em Clínica Médica, HCPA/UFRGS.

4. Estudante de Medicina, Bolsista de Iniciação Científica, UFRGS.

5. Médico Cardiologista, PhD em Cardiologia, Serviço de Cardiologia, HCPA.

6. Médico Cardiologista, Serviço de Cardiologia, HCPA

7. Médico Radiologista, Serviço de Radiologia, HCPA.

8. Médica Pneumologista, Serviço de Pneumologia, HCPA.

9. Professor Adjunto, Médico Reumatologista, PhD em Clínica Médica, Chefe do Serviço de Reumatologia, HCPA/UFRGS.
}

Endereço para correspondência: Dr. Ricardo M. Xavier. Serviço de Reumatologia do Hospital de Clínicas de Porto Alegre. R. Ramiro Barcelos 2350/ sala 645, CEP 90035-003, Porto Alegre, RS. E-mail: rmaxavier@hcpa.ufrgs.br 
discriminar pacientes com e sem o desfecho combinado de HAP ou doença intersticial pulmonar em pacientes com duração de doença $\leq 5$ anos. Conclusões: A severidade das anormalidades da CPU está associada com dano em órgãos-alvo na ES. A CPU pode indicar a presença de doença pulmonar ativa (representada por áreas de opacidades em vidro-fosco na TCAR) na ES de duração relativamente curta.

Palavras-chave: esclerose sistêmica, capilaroscopia, alveolite fibrosante, hipertensão pulmonar, tomografia computadorizada.

\section{INTRODUÇÃO}

A esclerose sistêmica (ES) é uma doença caracterizada por disfunção vascular, fibrose cutâneo-visceral e auto-anticorpos circulantes. $\mathrm{O}$ acometimento pulmonar (por fibrose ou doença vascular) é atualmente a causa mais importante de mortalidade nessa doença ${ }^{(1)}$.

Entre as formas de avaliação da disfunção vascular que ocorre na doença, a capilaroscopia periungueal (CPU) se destaca como um método de fácil execução e não-invasivo. $\mathrm{Na}$ década de 70, Maricq ${ }^{(2,3)}$ sistematizou o estudo da capilaroscopia e denominou de 'padrão SD' (de scleroderma) as alterações tipicamente observadas em pacientes com esclerose sistêmica, dermatomiosite e doença mista do tecido conjuntivo: dilatação de capilares associada a áreas avasculares e perda da organização normal dos capilares. O padrão SD é observado em 83\% a 97\% dos pacientes com esclerose sistêmica ${ }^{(2-7)}$.

Diversos estudos tentaram associar a presença ou severidade de alterações capilaroscópicas com lesões em órgãosalvo na ES, obtendo resultados variáveis ${ }^{(4,5,8-18)}$. Nenhum desses estudos tentou associar os achados capilaroscópicos com alterações na tomografia computadorizada de alta resolução (TCAR) pulmonar, que é um método muito mais sensível e precoce do que a radiografia de tórax para a detecção de doença intersticial pulmonar ${ }^{(19)}$. A TCAR permite a detecção de áreas de opacidades em vidro-fosco, que representam regiões de alveolite fibrosante ativa que podem evoluir para fibrose pulmonar irreversível ${ }^{(20)}$.

O objetivo do presente estudo é caracterizar melhor as variáveis clínicas associadas às alterações capilaroscópicas e definir o valor da CPU em predizer a presença e atividade de lesões em órgãos internos na ES. Especial interesse foi voltado para a doença pulmonar, que foi avaliada através de provas de função pulmonar, ecocardiografia e TCAR. Considerando-se que a duração da doença parece ser um importante fator associado à severidade das anormalidades to discriminate between patients with and without the combined end-point of PAH or interstitial disease in patients with disease duration $\leq 5$ years. Conclusions: The severity of NCM abnormalities is associated with end-organ damage in SSC. NCM may indicate the presence of active pulmonary disease (represented by ground-glass opacities areas in HRCT) in SSc of relatively short duration.

Keywords: systemic sclerosis, capillaroscopy, fibrosing alveolitis, pulmonary hypertension, computed tomography.

observadas na CPU ${ }^{(17,21-23)}$ e com o objetivo de diminuir a influência da duração da disfunção vascular da ES na interpretação das alterações observadas na capilaroscopia, algumas análises foram adicionalmente realizadas em um subgrupo de pacientes com duração de doença $\leq 5$ anos.

\section{PACIENTES E MÉTODOS}

Pacientes: Foram avaliados prospectivamente 98 pacientes consecutivos com diagnóstico definitivo ou forte suspeita de ES, de acordo com a avaliação de reumatologistas experientes. Para inclusão no presente estudo, os pacientes deveriam enquadrar-se nos critérios do Colégio Americano de Reumatologia (American College of Rheumatology ACR) ${ }^{(24)}$ ou nos critérios sugeridos por LeRoy e Medsger para o diagnóstico de formas precoces de ES (evidência objetiva de fenômeno de Raynaud mais padrão SD na CPU ou auto-anticorpos específicos para ES; alternativamente, evidência subjetiva de fenômeno de Raynaud mais padrão SD na CPU e auto-anticorpos específicos para ES) ${ }^{(25)}$. Foram excluídos pacientes que apresentavam síndromes de sobreposição com outras doenças difusas do tecido conjuntivo, infecções agudas ou crônicas ativas, pacientes com diabetes melito insulino-dependente ou com diabetes de mais de cinco anos de evolução. Pacientes com diagnóstico definido de esclerose sistêmica (de acordo com os critérios do ACR) que desenvolveram miopatia inflamatória não foram excluídos da amostra. Todos os pacientes assinaram termo de consentimento informado.

Avaliação clínica: Os pacientes foram entrevistados e examinados pelo mesmo pesquisador, de acordo com um extenso questionário padronizado voltado para a avaliação dos órgãos-alvo da esclerose sistêmica. Utilizou-se o escore de Rodnan modificado para avaliação da extensão do acometimento cutâneo ${ }^{(26)}$. A severidade da esclerodactilia foi avaliada de acordo com a escala proposta por Brennan et al. (ausente: pele normal; leve: espessamento cutâneo leve; 
moderada: pele espessada e incapaz de ser pinçada; severa: pele espessada e incapaz de ser movida ${ }^{(26)}$. A severidade da dispnéia foi graduada de acordo com a escala da New York Heart Association. Os pacientes foram classificados de acordo com o subtipo de doença em três categorias: forma difusa cutânea (espessamento cutâneo no tronco e extremidades); forma limitada cutânea (espessamento cutâneo restrito às extremidades e/ou face); forma limitada (ausência de espessamento cutâneo) $)^{(25,27)}$. A duração da doença foi definida como o tempo desde o aparecimento do fenômeno de Raynaud ou dos sintomas cutâneos (o que houvesse surgido primeiro).

A realização da CPU ocorreu em média 14 dias após a avaliação inicial, sob condições controladas (após pelo menos 20 minutos em repouso num ambiente com temperatura mantida entre $20^{\circ} \mathrm{C}-25^{\circ} \mathrm{C}$ ), de acordo com as especificações descritas abaixo. Foram coletadas amostras de sangue (imediatamente após a realização da CPU) para hemograma, sorologia, velocidade de sedimentação globular (VSG) e testes bioquímicos.

Capilaroscopia periungueal: Os dígitos 2,3 , 4 e 5 de ambas as mãos foram examinados com lupa estereoscópica Stemi-2000 C (Zeiss) com tubo extensor para máquina fotográfica, permitindo aumentos de 6,5 a 65 vezes. Dígitos com traumatismos ou outras alterações que pudessem prejudicar sua avaliação foram excluídos do exame. A iluminação da área periungueal foi feita com fonte de luz fria (lâmpada halógena) com incidência de luz a $45^{\circ}$. O meio diafanizador utilizado foi óleo de imersão (óleo de cedro $)^{(6,28)}$. Pelos menos dois dígitos de cada paciente foram fotografados utilizando-se uma câmera Contax 167MT acoplada à lupa.

Todos os exames capilaroscópicos foram feitos por um examinador com larga experiência na técnica e que não tinha conhecimento dos dados clínicos ou resultados dos exames dos pacientes. A avaliação de todos os exames seguiu uma ficha padronizada, baseada no modelo proposto por Andrade et al. ${ }^{(29)}$. O grau de desvascularização foi avaliado semiquantitativamente de acordo com o escore avascular de Lee (0- ausência de deleção capilar, 1- uma ou duas áreas discretas de deleção, 2- mais de duas áreas discretas de deleção, 3- áreas confluentes de deleção) em cada dedo examinado. Área de deleção é uma região avascular definida pela ausência de mais de dois capilares sucessivos na fileira distal periungueal. A soma dos graus de deleção foi dividida pelo número de dedos examinados para obtenção do escore dos graus de deleção (EGD), representando o valor médio dos graus de deleção de todos os dedos examinados em cada paciente ${ }^{(17)}$. O número de hemorragias, ectasias (alças com diâmetro de cerca de 4 ou mais vezes o normal) e megacapilares (alças capilares gigantes, com diâmetro de cerca de 10 ou mais vezes o normal) contados em cada dedo foram individualmente somados e divididos pelo número de dígitos examinados, fornecendo o número médio da cada uma dessas alterações por dedo examinado ${ }^{(17,29)}$. A presença do padrão SD foi definida qualitativamente como a ocorrência de áreas avasculares e/ou a presença de capilares definidamente dilatados, de acordo com descrição de Maricq ${ }^{(3)}$.

Considerando-se que as anormalidades capilaroscópicas na esclerose sistêmica são representadas por graus variáveis de desvascularização e dilatação capilar e que áreas avasculares parecem surgir principalmente a partir da ruptura e obliteração de alças capilares que se tornaram progressivamente dilatadas ${ }^{(30)}$, a deleção e dilatação foram incorporadas em um escore combinado de severidade. Esse escore foi calculado simplesmente tomando o maior valor numérico entre as duas seguintes variáveis: escore dos graus de deleção ou número médio de megacapilares por dedo (valor máximo=3.0). Para fins de análise, pacientes com um escore combinado de severidade $\geq 1$ foram considerados como tendo alterações capilaroscópicas severas.

Sorologia: Foi avaliada a presença do fator antinuclear (FAN) e de anticorpos antitopoisomerase I (anti-Scl-70) através das técnicas de imunofluorescência indireta (utilizando-se como substrato células HEP-2) e enzima imunoensaio (QUANTA lite ${ }^{\circledR}$ Scl-70 ELISA, INOVA Diagnostics), respectivamente. FAN foi considerado positivo quando reagente em diluições $\geq 1: 80$. A presença de anticorpos anticentrômero foi determinada de acordo com a observação do padrão típico da fluorescência em células HEP-2.

Tomografia computadorizada de alta resolução pulmonar (TCAR): As imagens foram obtidas sem o uso de contraste endovenoso, com o paciente em posição supina e em apnéia após inspiração profunda. Os cortes tomográficos foram obtidos com janela adequada para visualização de parênquima pulmonar e pleura, a intervalos determinados pelo radiologista executor do exame. A presença e a extensão da doença intersticial pulmonar (infiltrado em vidro-fosco e faveolamento) foram avaliadas por dois radiologistas. Eles estimaram, em consenso, o percentual (arredondado para os 5\% mais próximos) de parênquima acometido por doença intersticial em cada um dos hemisférios pulmonares, de acordo com o método proposto por Staples et al. ${ }^{(31)}$. Os escores finais pulmonares foram obtidos pela média dos percentuais estimados em ambos os pulmões. 
Provas de função pulmonar: Todos os pacientes foram submetidos a espirometria e teste de capacidade difusional de monóxido de carbono (DLCO). Manobras para a mensuração da capacidade vital forçada (CVF) foram realizadas de acordo com as recomendações da American Thoracic Society ${ }^{(32)}$, sendo a melhor CVF obtida em três esforços utilizada para análise. A medida da DLCO foi realizada pela técnica de inspiração única, com valores corrigidos pela concentração de hemoglobina sangüínea. Os resultados são apresentados como percentuais de valores previstos para adultos normais da mesma idade e superfície corporal ${ }^{(33,34)}$. CVF e DLCO foram consideradas anormais quando $<80 \%$ e $<75 \%$ do valor previsto, respectivamente.

Ecocardiografia com Doppler: A estimativa da pressão sistólica da artéria pulmonar (PSAP) foi obtida pela soma do gradiente pressórico transtricúspide e da pressão estimada na veia cava inferior $(\mathrm{VCI})^{(35)}$. Pacientes com PSAP $\geq 40 \mathrm{mmHg}$ foram considerados como apresentando hipertensão arterial pulmonar (HAP) ${ }^{(36)}$.

Cintilografia de trânsito esofágico: Realizada com os pacientes em posição supina sob uma gama-câmera de amplo campo de visão. Os pacientes receberam $6 \mathrm{ml} \mathrm{de}$ água artificialmente marcada com $2 \mathrm{mCi}$ de ${ }^{99}$ tecnéciofitato. Após acionamento do computador para aquisição de dados (com contagens a cada um segundo), os pacientes foram orientados a deglutir todo o conteúdo da boca de uma única vez. Atividade residual $\geq 20 \%$ (com relação ao pico de atividade radioativa) após 15 segundos do início da deglutição foi considerada anormal e sugestiva de disfunção esofágica ${ }^{(37)}$.

Todas as avaliações clínicas e exames capilaroscópicos foram realizados de abril de 2000 a novembro de 2001. Os exames ecocardiográficos, radiológicos, cintilográficos e provas de função pulmonar foram realizados dentro de 6 meses da CPU, com poucas exceções. Nenhum dos examinadores tinha informações sobre os detalhes clínicos dos pacientes (exceto pelo fato de saberem que tinham esclerose sistêmica) ou resultados de outros exames. O estudo foi aprovado pelo Comitê de Ética em Pesquisa do Hospital de Clínicas de Porto Alegre antes do início de sua execução.

Análise estatística: Os dados foram analisados usando-se os programas EPI-INFO versão 6, SPSS para Windows versão 6 e Medcalc versão 6 . A análise da associação entre variáveis categóricas foi feita utilizando-se o teste do quiquadrado (com correção de Yates se necessário) ou teste exato de Fisher. Variáveis quantitativas foram testadas graficamente quanto à normalidade da distribuição (com curvas de probabilidade normal) e também usando-se o teste de ajustamento (goodness-of-fit) de KolmogorovSmirnov. A variável escore dos graus de deleção (EGD), que apresentou pequeno desvio da normalidade, foi submetida a transformação logarítmica $\left(\log _{10}[\mathrm{EGD}+1]\right)$, de forma a permitir a utilização de testes paramétricos (teste t de Student e análise de variância), sendo conseqüentemente apresentada como média geométrica e intervalo de confiança de 95\% (IC 95\%). Diante da ocorrência de heterogeneidade de variâncias (avaliada pelo teste de Levene) ou distribuição não-normal de variáveis, aplicaram-se testes não-paramétricos (Mann-Whitney e coeficiente de correlação de Spearman $\left.\left[\mathrm{r}_{\mathrm{s}}\right]\right)$. O controle para fatores de confusão foi realizado através de análise de covariância. Valores de $\mathrm{P} \leq 0,05$ foram considerados estatisticamente significativos. Todos os valores de $\mathrm{P}$ apresentados são bicaudais.

Curvas ROC (do inglês receiver operating characteristic) foram usadas para testar a habilidade da CPU em diferenciar pacientes com e sem dano visceral. A área sob a curva ROC é uma medida adequada para quantificar a capacidade discriminatória de um modelo diagnóstico (representando a acurácia do modelo), variando entre 0,5 e 1,0 (nenhuma e máxima capacidades discriminatórias, respectivamente) ${ }^{(38)}$. Intervalos de confiança de 95\% para as áreas sob as curvas também foram calculados.

Para avaliação simultânea da associação de diversas variáveis com o escore dos graus de deleção (após transformação logarítmica), foram elaborados modelos de regressão linear múltipla. A adequação aos pressupostos dos modelos de regressão linear múltipla (normalidade da variável dependente, relação linear das variáveis independentes com a dependente, homogeneidade de variâncias da variável dependente, distribuição normal dos valores residuais, ausência de interações) foi testada, sendo que os modelos apresentados adequaram-se a esses pressupostos.

\section{RESULTADOS}

Dos 98 pacientes inicialmente avaliados, 4 foram excluídos por não preenchimento dos critérios de inclusão e ou por preenchimento de critérios de exclusão. Outros 3 pacientes não realizaram capilaroscopia periungueal (um foi incapaz de submeter-se ao exame devido a contraturas nos quirodáctilos e outro faleceu logo após a primeira avaliação; um terceiro paciente não retornou para $\mathrm{CPU})$. A descrição dos 91 pacientes estudados (classificados de acordo com o preenchimento dos critérios do ACR para esclerose sistê- 
mica e subtipo de doença) encontra-se na tabela 1. Vinte e dois foram classificados como forma cutânea difusa, 55 tinham a forma cutânea limitada e 14 tinham a forma limitada de ES.

A comparação dos escores dos graus de deleção (EGD) de acordo com as características clínicas pode ser vista na tabela 2 . Houve correlação estatisticamente significativa do EGD com o escore cutâneo total $\left(\mathrm{r}_{\mathrm{S}}=0,59, \mathrm{P}<0,001\right)$, severidade da esclerodactilia $\left(r_{s}=0,68, P<0,001\right)$ e com o tempo desde o início do fenômeno de Raynaud $\left(\mathrm{r}_{\mathrm{s}}=0,35, \mathrm{P}=0,001\right)$ e idade $\left(r_{s}=0,35, P=0,001\right)$. Pacientes com doença cutânea difusa e cutânea limitada tinham maiores escores de deleção (EGD médio $=1,54$, IC $95 \%=1,13-2,02$ e EGD médio=1,41, IC $95 \%=1,25-1,58$, respectivamente) do que aqueles com a forma limitada de ES (EGD médio $=0.81$, IC 95\% $=0,58-1,08$ ) (análise de variância, $\mathrm{P}=0,002$ ).
Testaram-se também as relações entre as alterações em exames complementares e a severidade da desvascularização (tabela 3). O tempo desde o início do fenômeno de Raynaud (duração do FRy) correlacionou-se ao escore de deleção e a algumas lesões em órgãos-alvo, podendo, portanto, representar um fator de confusão na associação da agressividade da doença com as alterações capilaroscópicas. Em razão disso, nessa tabela são apresentados os valores de P "bruto" e ajustados para duração do FRy. As presenças de anticorpos anti-Scl-70 e de infiltrado em vidro-fosco na TCAR (representando áreas de doença pulmonar ativa) associaram-se positivamente ao escore dos graus de deleção, principalmente após ajuste para duração do FRy, sugerindo desvascularização mais severa nesses pacientes. A tendência à associação dos anticorpos anticentrômero com maiores graus de deleção desapareceu após ajuste para duração do FRy.

TABELA 1

CARACTERÍstiCAS DEMOgRÁFICAS E CLÍNICAS DOS PACIENTES, DE ACORDO COM O PREENCHIMENTO DOS CRITÉRIOS DO ACR PARA ESCLEROSE SISTÊMICA (PACIENTES DESIGNAdOS COMO ACR POSITIVOS OU NEGATIVOS).*

\begin{tabular}{|c|c|c|c|c|}
\hline & \multicolumn{2}{|c|}{ ACR positivos } & \multicolumn{2}{|c|}{ ACR negativos } \\
\hline Cor branca & $16(72,7)$ & $43(89,6)$ & $17(85,7)$ & 0,197 \\
\hline Idade $(\text { anos })^{\ddagger}$ & $48,8 \pm 11,1$ & $53,4 \pm 11,3$ & $47,8 \pm 12,4$ & 0,111 \\
\hline Esclerodactilia & $22(100)$ & $46(95,8)$ & $5(23,5)$ & $<0,001$ \\
\hline Cicatrizes puntiformes ou reabsorção distal digital & $20(90,9)$ & $41(85,4)$ & $2(9,5)$ & $<0,001$ \\
\hline Esclerodermia proximal ${ }^{\llbracket}$ & $22(100)$ & $44(91,7)$ & $6(28,6)$ & $<0,001$ \\
\hline Calcinoses & $5(22,7)$ & $20(41,7)$ & $0(0,0)$ & 0,001 \\
\hline Telangiectasias & $13(59,1)$ & $29(60,4)$ & $5(23,8)$ & 0,014 \\
\hline FAN em título $\geq 1: 80$ & $19(86,4)$ & $43(89,6)$ & $15(71,4)$ & 0,152 \\
\hline Anticorpos anticentrômero\# & $4(19,0)$ & $25(54,3)$ & $7(33,3)$ & 0,017 \\
\hline Anticorpos anti-Scl-70\# & $6(28,6)$ & $9(19,6)$ & $2(9,5)$ & 0,294 \\
\hline Faveolamento ou infiltrado em vidro-fosco na TCAR\# & $13(68,4)$ & $23(52,3)$ & $8(38,1)$ & 0,122 \\
\hline Redução da CVF & $13(59,1)$ & $19(39,6)$ & $5(23,8)$ & 0,061 \\
\hline Redução da DLCO\# & $17(89,5)$ & $43(89,6)$ & $13(61,9)$ & 0,013 \\
\hline Disfunção esofágica\# & $17(81,0)$ & $41(85,4)$ & $12(57,1)$ & 0,031 \\
\hline Hipertensão arterial pulmonar\# & $2(9,1)$ & $9(19,1)$ & $1(4,8)$ & 0,217 \\
\hline
\end{tabular}

${ }^{*} 0$ s dados são apresentados como número (percentagem) de pacientes, exceto quando indicado. ${ }^{\dagger}$ Qui-quadrado, análise de variância ou teste de Kruskal-Wallis. ${ }^{\ddagger}$ Dados apresentados como média e desvio-padrão. ${ }^{\S}$ Dados apresentados como mediana, percentís 25 e 75. "Espessamento cutâneo afetando regiões proximais às articulações metacarpofalangeanas ou metatarsofalangeanas ou face. "Dados não disponíveis para alguns pacientes, sendo os percentuais calculados a partir de total de pacientes que realizaram os exames. 
TABELA 2

ComparaÇão dos escores dos Graus de deleÇÃo (EGD) DE ACORdo COM A PRESENÇA de ALTERAÇÕES CLÍNICAS EM PACIENTES COM ES (VALORES APRESENTADOS COMO MÉDIAS GEOMÉTRICAS E INTERVALOS DE CONFIANÇA DE 95\%).

\begin{tabular}{|c|c|c|c|c|c|}
\hline & & Presente(s) & & Ausente(s) & \\
\hline & $\mathrm{N}$ & Média (IC 95\%) & $\mathrm{N}$ & Média (IC 95\%) & $P^{*}$ \\
\hline Idade $\geq 50$ anos & 43 & $1,63(1,42-1,86)$ & 48 & $1,10(0,92-1,29)$ & $<0,001$ \\
\hline Cicatrizes puntiformes digitais & 46 & $1,68(1,48-1,89)$ & 45 & $1,03(0,86-1,22)$ & $<0,001$ \\
\hline Reabsorção distal digital & 56 & $1,51(1,30-1,75)$ & 35 & $1,08(0,92-1,24)$ & $0,001^{\dagger}$ \\
\hline Amputações digitais & 11 & $2,09(1,63-2,62)$ & 80 & $1,25(1,10-1,40)$ & 0,001 \\
\hline Calcinoses & 25 & $1,72(1,38-2,10)$ & 66 & $1,21(1,05-1,37)$ & 0,004 \\
\hline Telangiectasias & 47 & $1,60(1,37-1,84)$ & 44 & $1,09(0,92-1,27)$ & $<0,001$ \\
\hline Esclerodactilia & 73 & $1,46(1,29-1,65)$ & 18 & $0,89(0,68-1,11)$ & $<0,001$ \\
\hline Esclerodermia proximal ${ }^{\ddagger}$ & 72 & $1,49(1,32-1,68)$ & 19 & $0,83(0,65-1,02)$ & $<0,001$ \\
\hline Escore cutâneo $\geq 16$ & 18 & $2,21(1,95-2,50)$ & 73 & $1,16(1,02-1,31)$ & $<0,001$ \\
\hline Duração de $F R y \geq 10$ anos $^{\S}$ & 42 & $1,56(1,33-1,81)$ & 49 & $1,16(0,98-1,36)$ & 0,009 \\
\hline Preenchimento dos critérios do ACR & 70 & $1,49(1,31-1,68)$ & 21 & $0,89(0,70-1,09)$ & $<0,001$ \\
\hline
\end{tabular}

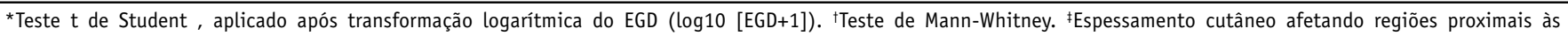

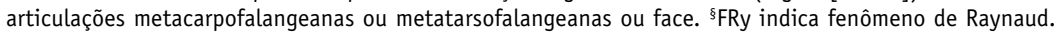

TABELA 3

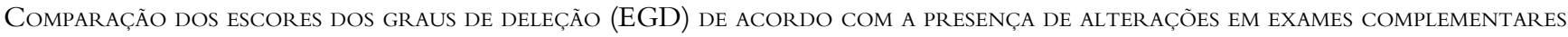
(VALORES APRESENTADOS COMO MÉDIAS GEOMÉTRICAS E INTERVALOS DE CONFIANÇA DE 95\%).

\begin{tabular}{|c|c|c|c|c|c|c|}
\hline & & Presente(s) & & Ausente(s) & & \\
\hline & $\mathrm{N}$ & Média (IC 95\%) & $\mathrm{N}$ & Média (IC 95\%) & $P^{*}$ & $P_{\text {ajust }}^{\dagger}$ \\
\hline Anemia & 21 & $1,60(1,21-2,05)$ & 70 & $1,26(1,11-1,43)$ & 0,073 & 0,107 \\
\hline VSG elevado & 24 & $1,31(0,97-1,70)$ & 61 & $1,35(1,19-1,52)$ & 0,801 & \\
\hline FAN $\geq 1: 80$ & 77 & $1,43(1,28-1,59)$ & 14 & $0,88(0,51-1,33)$ & 0,004 & 0,007 \\
\hline Anticorpos anticentrômero & 36 & $1,49(1,27-1,73)$ & 52 & $1,21(1,01-1,42)$ & 0,076 & 0,479 \\
\hline Anticorpos anti-Scl-70 & 17 & $1,73(1,39-2,11)$ & 71 & $1,23(1,07-1,40)$ & 0,016 & 0,003 \\
\hline CVF reduzida & 37 & $1,45(1,25-1,67)$ & 54 & $1,26(1,06-1,48)$ & 0,224 & \\
\hline DLC0 reduzida & 73 & $1,37(1,21-1,55)$ & 15 & $1,10(0,74-1,54)$ & 0,164 & \\
\hline Dismotilidade esofágica & 70 & $1,42(1,26-1,59)$ & 20 & $1,03(0,71-1,42)$ & 0,026 & 0,038 \\
\hline Faveolamento pulmonar & 27 & $1,44(1,18-1,73)$ & 59 & $1,32(1,14-1,53)$ & 0,509 & \\
\hline Opacidades em vidro-fosco & 43 & $1,53(1,32-1,75)$ & 43 & $1,20(0,99-1,44)$ & 0,040 & 0,002 \\
\hline Hipertensão arterial pulmonar & 12 & $1,62(1,24-3,00)$ & 78 & $1,29(1,13-1,46)$ & 0,162 & \\
\hline \multicolumn{7}{|l|}{ Alterações ecocardiográficas } \\
\hline valvulopatia & 20 & $1,58(1,25-1,95)$ & 70 & $1,26(1,10-1,44)$ & 0,096 & 0,602 \\
\hline dilatação de cavidades & 27 & $1,57(1,25-1,94)$ & 63 & $1,23(1,07-1,40)$ & 0,049 & 0,086 \\
\hline
\end{tabular}

*Teste $t$ de Student, aplicado após transformação logarítmica do EGD (log10 [EGD+1]). ${ }^{\dagger}$ Análise de covariância, com valor $\mathrm{P}$ ajustado para a duração do fenômeno de Raynaud (aplicada somente quando alguma tendência estatística era encontrada na análise bivariada).

A tabela 4 mostra um modelo de regressão linear múltipla tendo como variável dependente o escore dos graus de deleção (EGD), após ser submetido à transformação logarítmica. Observa-se que o escore cutâneo total, a duração do FRy, a presença de cicatrizes puntiformes digitais ou amputações em dedos, a presença de opacidades em vidro-fosco na TCAR e idade $\geq 50$ anos associaram-se significativa e independentemente com o EGD. A variável severidade da esclerodactilia perdeu significância estatística e relevância no modelo após ajuste para a influência das outras variáveis. O coeficiente de determinação do modelo sugere que aproximadamente $60 \%$ da variância do EGD 
TABELA 4

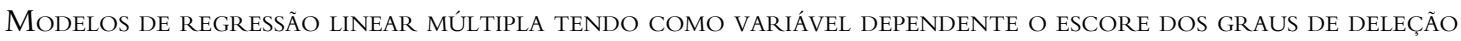
APÓS TRANSFORMAÇÃO LOGARÍTMICA (LOG 10 [EGD + 1]).

\begin{tabular}{|c|c|c|c|c|}
\hline Variáveis independentes & $B^{*}$ & $(\mathrm{IC} 95 \%)^{\dagger}$ & $\beta^{\ddagger}$ & $P$ \\
\hline Escore cutâneo total & 0,0062 & $(0,0040-0,0083)$ & 0,43 & $<0,001$ \\
\hline Cicatrizes puntiformes digitais ou amputações em dedos ${ }^{\S}$ & 0,0647 & $(0,0229-0,1065)$ & 0,24 & 0,003 \\
\hline Opacidades em vidro-fosco $0^{\S}$ & 0,0602 & $(0,0207-0,0997)$ & 0,22 & 0,003 \\
\hline
\end{tabular}

$R^{2}=0,60 ; R^{2}$ ajustado=0,57; $P<0,001$

\begin{tabular}{lcrrr}
\hline \multicolumn{1}{c}{ Pacientes com duração de doença $\leq 5$ anos $(\mathrm{N}=27)$} & \\
\hline Escore cutâneo total & 0,0061 & $(0,0019-0,0103)$ & 0,44 & 0,006 \\
Opacidades em vidro-fosco ${ }^{\S}$ & 0,1341 & $(0,0516-0,2167)$ & 0,49 & 0,003 \\
Constante & 0,1853 & $(0,1216-0,2492)$ & -- & $<0,001$
\end{tabular}

$R^{2}=0,51 ; R^{2}$ ajustado $=0,47 ; P<0,001$

*Coeficiente parcial de regressão. `Intervalo de confiança de $95 \%$ do coeficiente parcial de regressão. ${ }^{\ddagger}$ Coeficiente de correlação parcial. ${ }^{\S}$ Definido como ausente (0) ou presente (1).

(após transformação logarítmica) pode ser prevista por esse conjunto de variáveis. A aplicação do mesmo modelo em pacientes com 5 anos ou menos de doença mostrou que a presença de opacidades em vidro-fosco é o principal fator associado à deleção capilar nesses pacientes.

Análises semelhantes às apresentadas acima também foram realizadas tomando-se como variáveis dependentes o número médio de megacapilares, ectasias e hemorragias periungueais por dedo examinado. Pacientes com anticorpos anticentrômero apresentaram número significativamente maior de megacapilares (teste de MannWhitney, $\mathrm{P}=0,002)$, assim como pacientes com FAN positivo $(\mathrm{P}=0,010)$. Em pacientes com duração de doença $\leq 5$ anos, a presença do desfecho combinado de doença intersticial (opacidades em vidro-fosco ou faveolamento pulmonar na TCAR) ou hipertensão arterial pulmonar associou-se a um maior número de megacapilares $(\mathrm{P}=0,030)$, assim como a presença de disfunção esofágica $(\mathrm{P}=0,015)$. $\mathrm{O}$ número de hemorragias periungueais e ectasias não apresentou nenhuma associação clínica relevante.

A comparação das prevalências de dano visceral de acordo com a severidade das alterações capilaroscópicas é apresentada na tabela 5. Observa-se prevalência significativamente maior de doença esofágica e doença pulmonar ativa nos pacientes com alterações severas na CPU. Nenhum paciente com alterações capilaroscópicas leves ou com CPU normal apresentou acometimento por doença intersticial pulmonar (área total acometida por faveolamento e/ou infiltrado em vidro-fosco) superior a $5 \%$ do parênquima ou hipertensão arterial pulmonar (HAP).

A tabela 6 apresenta análises semelhantes à tabela 5 , porém restritas a pacientes com duração de doença $\leq 5$ anos. Observa-se forte associação da severidade das alterações capilaroscópicas com a presença de opacidades em vidrofosco. A sensibilidade da severidade das alterações na CPU para o desfecho combinado de doença intersticial ou hipertensão arterial pulmonar foi $100 \%$ (IC $95 \%=75,9-100$ ) e a especificidade foi $72,7 \%$ (IC $95 \%=39,3-92,7$ ). A análise restrita a pacientes preenchendo os critérios do ACR para a doença forneceu resultados semelhantes aos acima, mantendo-se a significância estatística para a associação entre doença pulmonar (intersticial ou HAP) e alterações severas na $\mathrm{CPU}(\mathrm{P}=0,002)$.

Considerando-se que a seleção de pontos de corte é um tanto arbitrária e pode produzir resultados "falsamente" significativos, testes adicionais da capacidade discriminatória do escore combinado de severidade foram feitos utilizandose curvas ROC. Estudando-se toda a amostra, esse escore mostrou fraca habilidade discriminatória (áreas sob as curvas ROC $<0,70)$ para a presença de opacidades em vidro-fosco (OVF) e para o desfecho combinado de doença intersticial 
TABELA 5

COMPARAÇÃO DAS PREVALÊNCIAS DE ANORMALIDADES ESOFÁGICAS E PULMONARES DE ACORDO COM A PRESENÇA DE ALTERAÇÕES SEVERAS

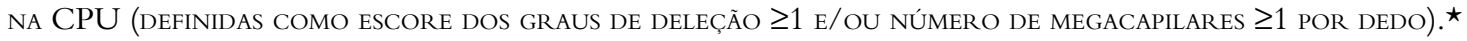

\begin{tabular}{lccc}
\hline & \multicolumn{1}{c}{ Alterações severasna CPU } & Não & $\mathrm{P}^{\dagger}$ \\
\hline Disfunção esofágica & Sim & $7 / 16(43,8)$ & 0,001 \\
Redução na CVF & $63 / 74(85,1)$ & $4 / 16(25,0)$ & 0,261 \\
Redução na DLCO & $33 / 75(44,0)$ & $10 / 16(62,5)$ & 0,026 \\
Hipertensão arterial pulmonar (HAP) & $63 / 72(87,5)$ & $0 / 16(0,0)$ & 0,114 \\
Opacidades em vidro-fosco & $12 / 74(16,2)$ & $2 / 13(15,4)$ & 0,016 \\
Faveolamento pulmonar & $41 / 73(56,2)$ & $1 / 13(7,7)$ & 0,055 \\
Doença intersticial ${ }^{\ddagger}$ ou HAP & $26 / 73(35,6)$ & $2 / 13(15,4)$ & 0,001 \\
\hline${ }^{*}$ Os val
\end{tabular}

${ }^{*}$ Os valores apresentados são o número de pacientes com as anormalidades indicadas sobre o total de pacientes examinados, com percentuais colocados entre parênteses.

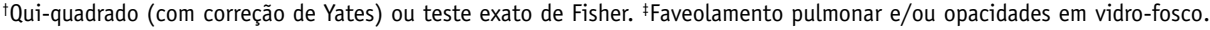

TABELA 6

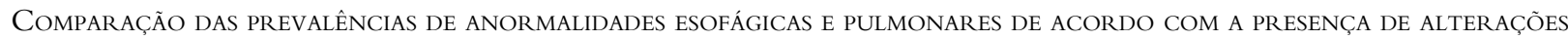
SEVERAS NA CPU (DEFINIDAS COMO ESCORE DOS GRAUS DE DELEÇÃo $\geq 1$ E/OU NÚMERO DE MEGACAPILARES $\geq 1$ POR DEDO) EM PACIENTES COM DURAÇÃO DE DOENÇA $\leq 5$ ANOS. *

\begin{tabular}{lccc}
\hline & \multicolumn{1}{c}{ Alterações severasna CPU } & Não & $\mathrm{P}^{\dagger}$ \\
\hline Disfunção esofágica & $\operatorname{Sim}$ & $4 / 10(40,0)$ & 0,044 \\
Redução na CVF & $16 / 20(80,0)$ & $2 / 10(20,0)$ & 0,119 \\
Redução na DLCO & $11 / 20(55,0)$ & $7 / 10(70,0)$ & 0,632 \\
Hipertensão arterial pulmonar (HAP) & $16 / 19(84,2)$ & $0 / 10(0,0)$ & 0,540 \\
Opacidades em vidro-fosco & $2 / 20(10,0)$ & $0 / 8(0,0)$ & $<0,001$ \\
Faveolamento pulmonar & $14 / 19(73,7)$ & $0 / 8(0,0)$ & 0,068 \\
Doença intersticial ${ }^{\ddagger}$ ou HAP & $7 / 19(36,8)$ & $0 / 8(0,0)$ & $<0,001$ \\
\hline
\end{tabular}

${ }^{\star}$ Os valores apresentados são o número de pacientes com as anormalidades indicadas sobre o total de pacientes examinados, com percentuais colocados entre parênteses. ${ }^{\dagger}$ Teste exato de Fisher. ¥Faveolamento pulmonar e/ou opacidades em vidro-fosco.

ou hipertensão arterial pulmonar (HAP). Entretanto, em pacientes com duração de doença $\leq 5$ anos, as áreas sob as curvas ROC foram 0,83 (IC $95 \%=0,63-0,94$ ) para OVF e 0,90 (IC 95\%=0,73-0,98) para o desfecho combinado de doença intersticial ou HAP (figura 1A), sugerindo uma boa capacidade discriminatória do escore combinado de severidade. Nesse mesmo subgrupo de pacientes, o escore dos graus de deleção (EGD) também mostrou boa habilidade discriminatória para OVF e para o desfecho combinado de doença intersticial ou HAP, com áreas sob as curvas ROC de 0,81 (IC 95\%=0,61-0,93) e 0,89 (IC 95\%=0,71-0,97), respectivamente. No entanto, o EGD demonstrou menor sensibilidade (mas maior especificidade) do que o escore combinado de severidade (figura 1B). O número médio de megacapilares por dedo mostrou uma razoável capacidade discriminatória para o desfecho combinado de doença intersticial ou HAP (área sob a curva ROC de 0,75, IC $95 \%=0,54-0,89)$ em pacientes com duração de doença $\leq 5$ anos.

A habilidade discriminatória de outras variáveis clínicas (escore cutâneo total, preenchimento dos critérios do ACR, forma difusa de doença, presença de esclerodermia proximal, presença e severidade de dispnéia e estertores crepitantes pulmonares, capacidade difusional pulmonar e anticorpos anti-Scl-70) também foi testada no subgrupo com duração de doença $\leq 5$ anos. Exceto para capacidade difusional pulmonar (área sob a curva ROC de 0,70, IC 95\%=0,49-0,86), nenhuma dessas variáveis apresentou capacidade discriminatória razoável para o desfecho combinado de doença intersticial ou hipertensão arterial pulmonar. Para a presença de opacidades em vidro-fosco, nenhuma das variáveis mostrou capacidade discriminatória significativa. 


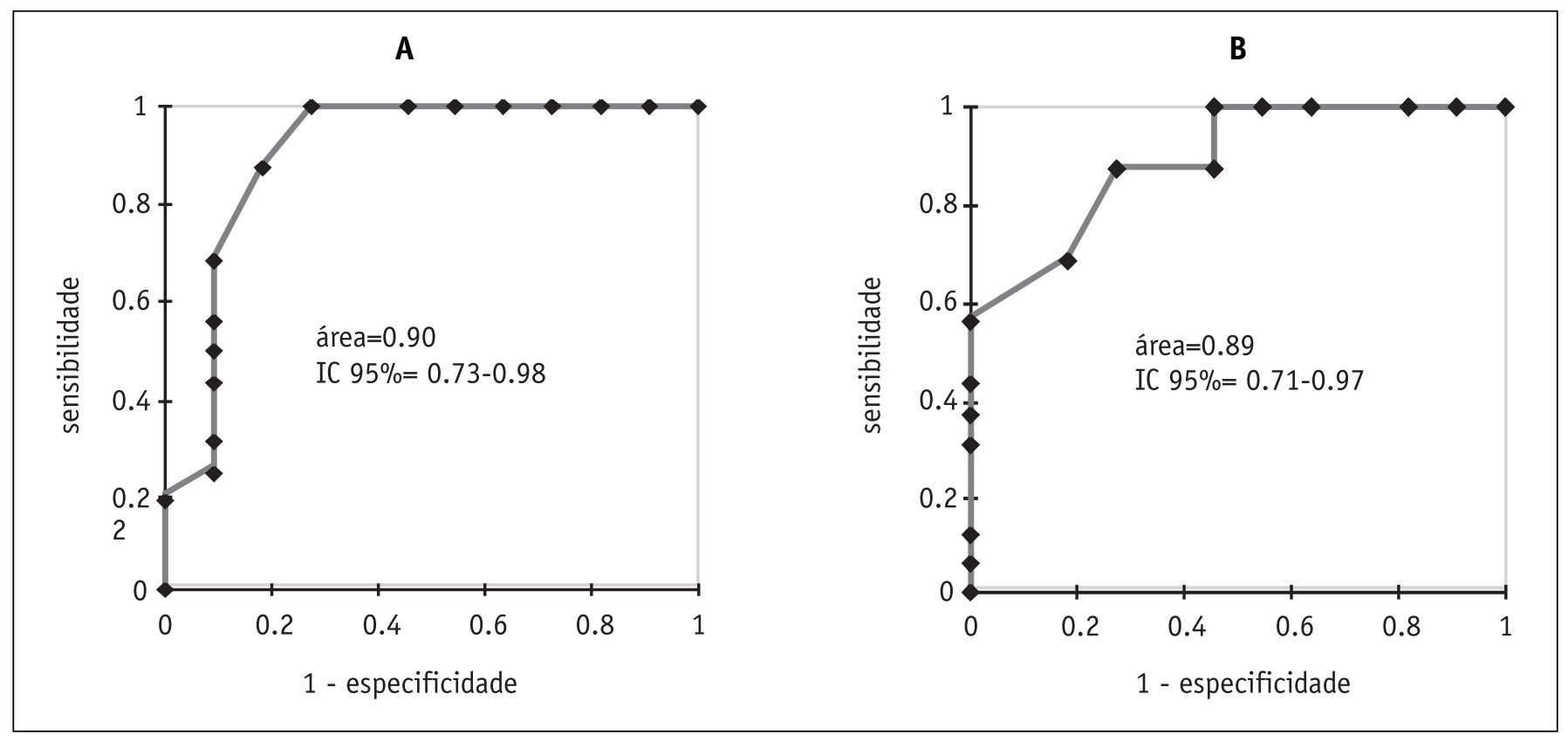

FigurAs 1A e 1B - Curvas ROC do escore combinado de severidade (A) e do escore dos graus de deleção (B) para o desfecho combinado de doença intersticial ou hipertensão arterial pulmonar em pacientes com duração de doença $\leq 5$ anos.

\section{DISCUSSÃO}

Os resultados apresentados demonstram estreita associação entre alterações capilaroscópicas, especialmente desvascularização, e extensão das lesões cutâneas na ES, conforme observado previamente ${ }^{(4,11,13,16,23,39)}$. A duração do fenômeno da Raynaud também foi significativamente associada à piora na desvascularização, independente de outras variáveis. Esse achado, corroborado por alguns trabalhos ${ }^{(17,21-23)}$, sugere que o dano vascular contínuo e prolongado inerente à doença seja um fator relevantemente associado às alterações vasculares observadas na CPU. Como a idade foi um fator independente associado a uma maior desvascularização, é possível que a suscetibilidade ao dano vascular seja maior em pacientes mais idosos.

Achados relacionados à presença dos auto-anticorpos indicam que esses podem ser um importante fator relacionado às alterações qualitativas e quantitativas da CPU. Estudos prévios associaram a presença de anticorpos anticentrômero a um padrão capilaroscópico onde há predomínio de megacapilares (às vezes denominado padrão SD lento em razão da pouca modificação do quadro vascular em exames repetidos $)^{(6,7,13)}$. Esses achados são corroborados pelo presente estudo, que observou um maior número de alças capilares gigantes em pacientes com anticorpos anticentrômero. Por outro lado, o estudo de fotografias periungueais seriadas realizado por Wong et al. ${ }^{(30)}$ sugere que as áreas avasculares na CPU são derivadas principalmente da ruptura, extravasamento e obliteração de alças capilares que previamente sofreram dilatação progressiva. A análise conjunta desses dados sugere que nos pacientes com anticentrômero a evolução do dano vascular ocorre de maneira mais lenta, com os megacapilares tendo maior "tempo de sobrevida" antes de sofrerem obstrução ou mesmo persistindo indefinidamente sem sofrerem obliteração, permitindo a observação de um maior número desses capilares. A presença do anti-Scl-70 associou-se à maior desvascularização, reproduzindo resultados obtidos em estudo prévio ${ }^{(5)}$. A explicação para esse fenômeno não é clara, mas a associação do anti-Scl-70 com acometimento cutâneo e visceral mais severo pode também representar dano vascular mais acentuado e precoce.

Apesar de diversos estudos relatarem associação da dilatação capilar ou desvascularização na CPU com lesões em órgãos internos ${ }^{(5,8-16)}$, outros falharam em reproduzir esses achados $^{(4,17,18,22)}$. A ampla variabilidade nos resultados devese possivelmente a diferenças amostrais e a diferentes métodos de aferição de alterações clínicas e da CPU. Maricq et al. ${ }^{(7)}$ publicaram um dos poucos estudos relacionando alterações na CPU à atividade de doença em pacientes com padrão SD, 
sugerindo que pacientes com padrão SD 'ativo' (com predomínio de desvascularização) mostraram progressão de doença, enquanto aqueles com padrão SD 'lento' apresentaram, em sua maioria, doença estacionária. Até onde sabemos, somente um estudo prévio testou, em uma análise secundária, a associação entre parâmetros de atividade de doença pulmonar e severidade das alterações capilaroscópicas. Silver et al. ${ }^{(40)}$, realizando lavado bronquíolo-alveolar (LBA) em pacientes com ES, observaram que pacientes com LBA com perfil inflamatório tenderam a ter maior prevalência de padrão SD na CPU do que pacientes com LBA não-inflamatório $(8 / 8$ versus $4 / 7$, respectivamente; $\mathrm{P}=0,077)$. $\mathrm{O}$ número de pacientes estudados foi muito pequeno para permitir a obtenção de resultados mais conclusivos.

No presente estudo, que conta com uma amostra relativamente grande de pacientes com ES, o uso da tomografia computadorizada de alta resolução pulmonar (TCAR) permitiu avaliar a presença, extensão e atividade da doença intersticial pulmonar. Na TCAR, áreas de opacidades em vidro-fosco (OVF) representam regiões de infiltrado inflamatório ativo ${ }^{(41)}$, podendo subseqüentemente evoluir para faveolamento, que representa fibrose pulmonar irreversível ${ }^{(20)}$. Utilizando-se métodos de análise multivariada, observou-se que existe associação entre a presença de OVF e a severidade da desvascularização na CPU, mesmo após ajuste para vários potenciais fatores de confusão. Nos pacientes com duração de doença $\leq 5$ anos, que representam um subgrupo que sofreu menor exposição temporal ao dano vascular associado à ES, a variável mais fortemente associada ao escore de deleção foi a presença de OVF na tomografia pulmonar de alta resolução. Os resultados também sugerem que a severidade das alterações capilaroscópicas pode ter boa capacidade discriminatória na avaliação da presença e atividade de doença pulmonar na esclerose sistêmica de evolução relativamente curta. Nenhuma outra variável (derivada do exame clínico ou exames complementares) foi comparável à CPU nesse contexto.

Uma questão que pode ser levantada é por que a CPU teria boa capacidade discriminatória para atividade de doença pulmonar somente em pacientes com duração relativamente curta de doença. Como demonstrado por esse e outros estudos, a duração de doença é um fator preditivo independente da perda de capilares, significando provavelmente que mesmo pacientes com doença leve ou de lenta evolução (mas de longa duração) podem desenvolver desvascularização severa após muitos anos de doença. Por outro lado, um número elevado de alças capilares gigantes em pacientes com duração curta de doença pode representar uma rápida evolução da deleção capilar (e doença mais agressiva) nesses pacientes, considerando-se que áreas avasculares parecem originar-se predominantemente a partir da obliteração de alças capilares que sofreram dilatação progressiva. No entanto, em pacientes com longa duração de doença, um número elevado de alças capilares gigantes provavelmente está relacionado à evolução lenta das alterações capilaroscópicas e clínicas. Esses fatores podem estar associados a uma redução significativa da especificidade da CPU para atividade de doença pulmonar em pacientes com longa evolução da doença.

A capilaroscopia periungueal não pôde diferenciar adequadamente pacientes com e sem padrão restritivo ou capacidade difusional reduzida em provas de função pulmonar. A presença de padrão restritivo e de capacidade difusional reduzida pode representar formas iniciais de doença intersticial e/ou vascular pulmonar, podendo ser constatados em pacientes com nenhuma ou discretas alterações na TCAR e níveis de pressão arterial pulmonar dentro de valores normais. Considerando-se também que pacientes com alveolite fibrosante ativa (detectada principalmente por lavado bronquíolo-alveolar) podem ter TCAR normal ${ }^{(42,43)}$, é possível que alguns pacientes com doença pulmonar ativa não tenham sido adequadamente identificados.

Diante dos resultados obtidos e dos dados disponíveis na literatura, conclui-se que a extensão da desvascularização na CPU está fortemente associada com a severidade, atividade e duração da esclerose sistêmica. Mais importante do ponto de vista clínico, a CPU pode indicar a presença de doença pulmonar ativa em pacientes com duração relativamente curta da doença. Considerando-se que a presença de doença pulmonar ativa está associada a uma boa resposta ao tratamento imunossupressor ${ }^{(44)}$, os resultados sugerem que a capilaroscopia periungueal pode auxiliar na seleção de pacientes que necessitam avaliação mais extensa e intervenções terapêuticas mais agressivas.

\section{AGRADECIMENTOS}

Agradecemos a Flávio Danni Fuchs, Dolores H. C. Ludwig, Raquel Faccioni, Marcus Franck, Tatiana Freitas Tourinho, Marcelo Maltchick, Paulo Sérgio Thys, Patrícia Minuzzi da Motta, Max Brenner, Tamara Mucenic, Adriano Barbiero, Tatiana Karenini Müller, Carmen Both 
Schenatto, Charles Lubianca Kohem, Lilian ScusselLonzetti e Ilóite Scheibel o seu valoroso auxílio. Também agradecemos a Eliane Regina Moreira Pereira, Janie Pires

\section{REFERÊNCIAS}

1. Black CM, du Bois RM: Organ involvement: pulmonary. In: Clements PJ, Furst DE, eds. Systemic Sclerosis. $1^{\text {a }}$ edição, Baltimore, Williams and Wilkins, págs. 299-331, 1996.

2. Maricq HR, LeRoy EC: Patterns of finger capillary abnormalities in connective tissue disease by "wide-field" microscopy. Arthritis Rheum 16: 619-28, 1973.

3. Maricq HR, LeRoy EC, D'Angelo WA, et al: Diagnostic potential of in vivo capillary microscopy in scleroderma and related disorders. Arthritis Rheum 23: 183-9, 1980.

4. Simeon CP, Fonollosa V, Vilardell M, et al: Study of the capillary microscopy changes in scleroderma and their association with organ disease, clinical manifestations and disease progression. Med Clin (Barc) 97: 561-4, 1991.

5. Schmidt KU, Mensing $\mathrm{H}$ : Are nailfold capillary changes indicators of organ involvement in progressive systemic sclerosis? Dermatologica 176: 18-21, 1988.

6. Carpentier PH, Maricq HR: Microvasculature in systemic sclerosis. Rheum Dis Clin North Am 16: 75-91, 1990.

7. Maricq HR, Harper FE, Khan MM, Tan EM, LeRoy EC: Microvascular abnormalities as possible predictors of disease subsets in Raynaud phenomenon and early connective tissue disease. Clin Exp Rheumatol 1:195-205, 1983.

8. Maricq HR, Spencer-Green G, LeRoy EC: Skin capillary abnormalities as indicators of organ involvement in scleroderma (systemic sclerosis), Raynaud's syndrome and dermatomyositis. Am J Med 61: 862-70, 1976.

9. Ohtsuka T, Ishikawa H: Statistical definition of nailfold capillary pattern in patients with systemic sclerosis. Dermatology 188: 286-9, 1994.

10. Ohtsuka T, Hasegawa A, Nakano A, Yamakage A, Yamaguchi M, Miyachi Y: Nailfold capillary abnormality and pulmonary hypertension in systemic sclerosis. Int J Dermatol 36: 116-22, 1997.

11. Pucinelli ML, Atra E, Sato EI, Andrade LE: Nailfold capillaroscopy in systemic sclerosis: correlations with involvement of lung and esophagus. Rev Bras Reumatol 35: 136-42, 1995.

12. Groen H, Wichers G, ter Borg EJ, van der Mark TW, Wouda AA, Kallenberg CG: Pulmonary diffusing capacity disturbances are related to nailfold capillary changes in patients with Raynaud's phenomenon with and without an underlying connective tissue disease. Am J Med 89: 34-41, 1990.

13. Chen ZY, Silver RM, Ainsworth SK, Dobson RL, Rust P, Maricq HR: Association between fluorescent antinuclear antibodies, capillary patterns, and clinical features in scleroderma spectrum disorders. Am J Med 77: 812-22, 1984.

14. Houtman PM, Kallenberg CG, Wouda AA, The TH: Decreased nailfold capillary density in Raynaud's phenomenon: a reflection of immunologically mediated local and systemic vascular disease? Ann Rheum Dis 44: 603-9, 1985. dos Santos, Leila Krammer, Lorena Koglin, Denílson dos Santos Marques, Rossimar Rocha, Andréia da Silva Ramiro e Iara Salin Gonçalves.

15. Joyal F, Choquette D, Roussin A, Levington C, Senecal JL: Evaluation of the severity of systemic sclerosis by nailfold capillary microscopy in 112 patients. Angiology 43: 203-10, 1992.

16. The Genovese Group for the Study of Systemic Sclerosis: Clinical evaluation of systemic sclerosis: a comprehensive panel of diagnostic tests to assess skin, microvascular and visceral involvement. Ann Ital Med Int 14: 79-85, 1999.

17. Lee P, Leung FY, Alderdice C, Armstrong SK: Nailfold capillary microscopy in the connective tissue diseases: a semiquantitative assessment. J Rheumatol 10: 930-8, 1983.

18. Statham BN, Rowell NR: Quantification of the nail fold capillary abnormalities in systemic sclerosis and Raynaud's syndrome. Acta Derm Venereol 66:139-43, 1986.

19. Schurawitzki H, Stiglbauer R, Graninger W, et al: Interstitial lung disease in progressive systemic sclerosis: high-resolution CT versus radiography. Radiology 176: 755-9, 1990.

20. Terrif BA, Kwan SY, Chan-Yeung MM, Müller NL: Fibrosing alveolitis: chest radiography and $\mathrm{CT}$ as predictors of clinical an functional impairment at follow-up in 26 patients. Radiology 184: 445-9, 1992.

21. Cutolo M, Sulli A, Pizzorni C, Accardo S: Nailfold videocapillaroscopy assessment of microvascular damage in systemic sclerosis. J Rheumatol 27: 155-60, 2000

22. Lovy M, MacCarter D, Steigerwald JC: Relationship between nailfold capillary abnormalities and organ involvement in systemic sclerosis. Arthritis Rheum 28: 496-501, 1985.

23. Scussel-Lonzetti L, Joyal F, Raynauld JP, et al: Predicting mortality in systemic sclerosis: analysis of a cohort of 309 French Canadian patients with emphasis on features at diagnosis as predictive factors for survival. Medicine (Baltimore) 81: 154-67, 2000.

24. Subcommittee for Scleroderma Criteria of the American Rheumatism Association Diagnostic and Therapeutic Criteria Committee: Preliminary criteria for the classification of systemic sclerosis (scleroderma). Arthritis Rheum 23: 581-90, 1980.

25. LeRoy EC, Medsger TA: Criteria for the classification of early systemic sclerosis. J Rheumatol 28: 1573-6, 2001.

26. Brennan P, Silman A, Black C, et al: Reliability of skin involvement measures in scleroderma. The UK Scleroderma Study Group. Br J Rheumatol 31: 457-60, 1992.

27. LeRoy EC, Black C, Fleischmajer R, et al: Scleroderma (systemic sclerosis): classification, subsets and pathogenesis. J Rheumatol 15 : 202-5, 1988.

28. Maricq HR: Widefield capillary microscopy: Techniche and rating scale for abnormalities seen in scleroderma and related disorders. Arthritis Rheum 24: 1159-65, 1981.

29. Andrade LE, Gabriel Junior A, Assad RL, Ferrari AJ, Atra E: Panoramic nailfold capillaroscopy: a new reading method and normal range. Semin Arthritis Rheum 20: 21-31, 1990 
30. Wong ML, Highton J, Palmer DG: Sequential nailfold capillary microscopy in scleroderma and related disorders. Ann Rheum Dis 47: 53-61, 1988.

31. Staples CA, Müller NL, Vedal S, Abboud R, Ostrow D, Miller RR: Usual Interstitial Pneumonia: Correlation of CT with Clinical, Functional, and Radiologic Findings. Radiology 162: 377-81, 1987.

32. American Thoracic Society: Lung function testing: selection of reference values and interpretative strategies. Am Rev Respir Dis 144: 1202-18, 1991.

33. Crapo RO, Morris AH: Standardized single breath normal values for carbon monoxide diffusing capacity. Am Rev Respir Dis 123: 185-9, 1981.

34. Crapo RO, Morris AH, Gardner RM: Reference spirometric values using techniques and equipment that meet ATS recommendations. Am Rev Respir Dis 123: 659-64, 1981.

35. Yock PG, Popp RL: Noninvasive estimation of right ventricular systolic pressure by Doppler ultrasound in patients with tricuspid regurgitation. Circulation 70: 657-62, 1984.

36. Murata I, Takenaka K, Yoshinoya S, et al: Clinical evaluation of pulmonary hypertension in systemic sclerosis and related disorders. A Doppler echocardiographic study of 135 Japanese patients. Chest 111: 36-43, 1997.

37. Tatsch K, Schroettle W, Kirsch CM: Multiple swallow test for the quantitative and qualitative evaluation of esophageal motility disorders. J Nucl Med 32: 1365-70, 1991.
38. Hanley JA, McNeil BJ: The meaning and use of the area under a receiver operating characteristic (ROC) curve. Radiology 143: 29-36, 1982.

39. Lee P, Sarkozi J, Bookman AA, Keystone EC, Armstrong SK: Digital blood flow and nailfold capillary microscopy in Raynaud's phenomenon. J Rheumatol 13: 564-9, 1986.

40. Silver RM, MetcalfJF, Stanley JH, LeRoy EC: Interstitial lung disease in scleroderma. Analysis by bronchoalveolar lavage. Arthritis Rheum 27: 1254-62, 1984.

41. Wells AU, Hansell DM, Corrin B, et al: High resolution computed tomography as a predictor of lung histology in systemic sclerosis. Thorax 47: 738-42, 1992

42. Wells AU, Hansell DM, Rubens MB, et al: Fibrosing alveolitis in systemic sclerosis. Bronchoalveolar lavage findings in relation to computed tomographic appearance. Am J Respir Crit Care Med 150: 462-8, 1994.

43. Dansin E, Wallaert B, Remy-Jardin M, et al. Study of highresolution thoracic computerized tomography and bronchoalveolar lavage in 36 patients presenting with systemic disease and a normal thoracic radiography. Rev Mal Respir 8: 551-8, 1991.

44. Wells AU, Hansell DM, Rubens MB, Cullinan P, Black CM, du Bois RM: The predictive value of appearances on thin-section computed tomography in fibrosing alveolitis. Am Rev Respir Dis 148: 1076-82, 1993. 